\title{
Biodynamic Response of Seated Human Body to Roll Vibration and Correlation between Roll and Lateral Directions
}

\author{
Zefeng Lin $\mathbb{D}^{1},{ }^{1}$ Junhong Zhang, ${ }^{1,2}$ Jinlu Li, ${ }^{1}$ Weitan Yin, ${ }^{3}$ Chi Liu $\mathbb{D}^{\circ},{ }^{3}$ and Jiewei Lin $\mathbb{D}^{1}$ \\ ${ }^{1}$ School of Mechanical Engineering, Tianjin University, Tianjin, China \\ ${ }^{2}$ Renai College, Tianjin University, Tianjin, China \\ ${ }^{3}$ Institute of Sound and Vibration Research, University of Southampton, Southampton, UK \\ Correspondence should be addressed to Chi Liu; liuchi0511@gmail.com and Jiewei Lin; linjiewei@tju.edu.cn
}

Received 11 May 2020; Accepted 6 October 2020; Published 3 November 2020

Academic Editor: Gerardo Silva-Navarro

Copyright (C) 2020 Zefeng Lin et al. This is an open access article distributed under the Creative Commons Attribution License, which permits unrestricted use, distribution, and reproduction in any medium, provided the original work is properly cited.

Within $30 \mathrm{~Hz}$, the discomfort caused by whole-body vibration in rotational direction is higher than vertical vibration at similar equivalent magnitude. Roll vibration, in particular, produces greater discomfort comparing with pitch and yaw vibrations. It is critical to understand the biodynamic characteristics of seated human body under roll vibration for both comfort assessment and vibration control. Experiments are carried out to obtain the biodynamic response of seated human body under random roll vibrations at four r.m.s. magnitude levels. It is found that the principal resonance in the roll apparent inertia is about $1 \mathrm{~Hz}$, but varied from 0.7 to $1.5 \mathrm{~Hz}$ depending on the magnitude of vibration $\left(0.5\right.$ to $\left.2.0 \mathrm{rad} / \mathrm{s}^{2}\right)$, and the secondary resonance locates around $3 \mathrm{~Hz}$ with a much lower modulus. It is noted that the human response to roll vibration has some features in common with that in the lateral direction. Two lumped parameter models are developed and calibrated to study the correlation between the two excitation axials. The equivalent relationships of magnitude and phase between roll and lateral vibrations are obtained on condition that they produce similar rotational responses of the upper human body. It suggests an equivalence approach between translational and rotational vibrations that can benefit the comfort assessment when exposed to multiaxial excitations.

\section{Introduction}

People are exposed to various vibrations from motorbike, car, subway, aircraft, ferry, and train during daily life transportations, which may cause health problems and discomfort. It is expected that the vehicles are well designed to provide better level of comfort or protection for occupants especially those who drive or ride vehicles for a long time in their daily routines [1]. However, analysis and improvement of ride comfort are a complex task, which was influenced by many factors such as the human biodynamic characteristics, the subjective response of human, and dynamic characteristics of vehicles and seats.

Biodynamic characteristics of human instruct the design of ride comfort of vehicles. To evaluate it, the frequency response functions such as mechanical impedance and apparent mass were used in experimental researches. $\mathrm{Wu}$ [2] indicated that apparent mass was more likely to reveal the inherent damped resonant frequency of the human body than impedance. The studies on apparent mass of seated human body exposed to vertical, lateral, and fore-and-aft vibration as well as the effect of the nonlinearity of human body and some parameters such as posture and thigh contact were reported. The first resonance frequency of vertical driving point apparent mass was observed in the range from 4 to $8 \mathrm{~Hz}$ and the second peak was seen between 9 and $15 \mathrm{~Hz}$ for most subjects [3-5]. The effects of posture, footrest, backrest, muscle tension, and vibration magnitude were investigated meanwhile. The nonlinearity of biodynamic response of seated human body was detected under excitations with different magnitudes. It was generally believed that the principal resonance frequency would decrease with increasing magnitude of vibration, which may be attributed to the "softening effect" of human body [6-9]. When it came to the horizontal response of human body, two heavily damped modes were detected for seated human body under 
lateral and fore-and-aft vibration without backrest [10]. The first resonance frequency was at about $0.7 \mathrm{~Hz}$ for both the fore-and-aft and the lateral directions and another peak in lateral apparent mass, which was not so pronounced, was observed around $2.5 \mathrm{~Hz}$. It was concluded that the first mode was dominated by the motion of rocking and swaying of the upper body. The effect of thigh contact, gender, and knee angle was investigated $[8,11-13]$. Based on the research with single-axial excitation, biodynamic responses to multiaxial vibration were reported [4, 14-17]. Considering the nonlinearity of human body, the linear superposition of the response under single-axial vibration may not be applied to the multiaxial case directly. Apart from frequency analysis basing on Fourier transform, the wavelet transfer function was proposed to evaluate human body response to translational excitation, in both frequency and time domain, which provided another perspective to evaluate biodynamic characteristics of human body [18].

The biodynamic responses of human include not only translational motion but also rotational motion. Current researches on human response in rotational direction are mostly excited by translational excitations, such as pitch response and roll response excited by vertical vibration $[8,19-22]$. Cross-axis apparent mass and transmissibility were widely used to describe this kind of response. It was found that, under vertical vibration, pitch motion of the pelvis also occurred at the resonance frequency while the pitch resonance of the pelvis occurred at higher frequencies, but the roll resonance was not detected in the range of $0-10 \mathrm{~Hz}[8,20]$. However, the vibrations which occupants are exposed to in vehicles are not just translational excitation. Rotational vibration is also very common, especially in roll and pitch directions. It is believed that roll vibration will produce greater discomfort than pitch and vertical vibration at the same level and similar frequency [23]. Beard [24-26] carried out researches on the subjective response of human body under roll vibration. Results showed that the roll vibration had a great influence on discomfort of seated human body. It indicates that understanding the biodynamic characteristics of human body under roll vibration is vital to the optimization of ride comfort in transportations. The seat-to-head transmissibility of human body under roll vibration was obtained by Paddan and Griffin [27]. The primary resonance was detected at $1.5 \mathrm{~Hz}$. It is not sufficient to characterise the rotational response of upper body under rotational excitation with seat-to-head transmissibility only while there are few reports on the measurements of other parameters which are similar to apparent mass or mechanical impedance. Depending on the rotational centre, the response in horizontal direction of the human body under roll excitations with the same magnitude may be different. That is to say, the horizontal cross-axis apparent mass is not sufficient for the description of human response to a rotational vibration. It is necessary to use the rotational motion parameters such as angular acceleration and torque to represent it. Besides, whether the roll motion caused by a lateral vibration and that caused by a roll excitation are the same is not known yet. If the correlation between roll and lateral excitation is confirmed, an equivalence method that can correlate the rotational and translational vibrations is essential and benefits the assessment of rotational response of human body to rotational vibration.

This study aims to obtain the dynamic characteristics of seated human body under roll vibration and find out the correlation of response to roll and lateral vibrations. Apparent inertia of seated human body under roll vibration is obtained by experiments with eight subjects. Two lumped parameter models sharing identical inertia and geometric parameters are developed and calibrated. After discussions on the biodynamic response to roll vibration, an equivalent approach to correlate the human responses under roll and lateral excitations is proposed.

\section{Experimental Method}

2.1. Design. Dynamic moment of inertia over the interface of seat pan and human body is obtained through the dynamic forces in vertical direction measured with a force plate (Kistler 9281B) and the horizontal distance between the sensors. Angular acceleration is acquired likewise with two SIT-pad accelerometers. Random roll vibrations produced by a 6-axis motion simulator are used as the excitation. The excitation signal has approximately flat constant bandwidth acceleration power spectrum defined in the range from 0.5 to $10 \mathrm{~Hz}$ with different magnitudes $\left(0.5,1.0,1.5\right.$, and $2.0 \mathrm{rad} / \mathrm{s}^{2}$ r.m.s. $)$. The rotation axis during the test is in the middle line of the seatsurface ( $x$-axis) following the definition in ISO 2631-1:1997. Every subject is exposed to the four levels of vibration for $66 \mathrm{~s}$, which comprised 4 seconds tamper before and after the formal test to obtain the dynamic response.

The frequency response function of seated human body under roll vibration, apparent inertia, is calculated by the moment of force and angular acceleration measured on the seat pan for characterising the biodynamic response. It is defined as the cross-spectrum of the torque and angular acceleration divided by the auto-spectrum of the angular acceleration, which is like

$$
I(f)=\frac{G_{a_{R} M_{R}}(f)}{G_{a_{R}}(f)},
$$

where $G_{a_{R}}(f)$ is the auto-spectrum of the angular acceleration about $x$-axis at the seat pan, $G_{a_{R} M_{R}}(f)$ is the crossspectrum between angular acceleration and torque about $x$ axis at the seat pan, and $I(f)$ is the apparent inertia. Similar to the apparent mass which is used to characterise the biodynamic response of human body to translation excitation, apparent inertia is applied when human body is subjected to rotation excitation. It represents the dynamic inertia of human body under roll excitation.

2.2. Apparatus. Roll vibrations are produced by a six-axis vibration simulator (Servotest Testing Systems Ltd., Surrey, $\mathrm{UK})$ with control and monitor system. In the experiments, subjects sit on a rigid seat $(0.56 \mathrm{~m}$ in height, $0.5 \mathrm{~m}$ in width, and $0.5 \mathrm{~m}$ in depth). A backrest, which subjects are not permitted to lean on during the test, is mounted on the rigid seat for safety. A safe belt and an emergency button are also 
provided. The rigidity of the seat has been verified before the experiment by measuring its transfer function whose modulus is always around 1 under $10 \mathrm{~Hz}$. The feet are supported by a footstep on the vibrator platform. The test setup is given in Figure 1.

The moment of force at the seat pan is measured by a force plate (Kistler 9281 B) with four transducers at four corners of the plate together with Kistler 566 charge amplifiers. The four force transducers are divided into two groups according to whether the sensor is on the left or right side of the force plate. Two sandbags with known mass of $5 \mathrm{~kg}$ for each (around $98 \mathrm{~N}$ ) are placed on the transducers, respectively, to have a group of sensors calibrated in vertical direction. The horizontal distance in $y$-direction of the two groups of transducers is $400 \mathrm{~mm}$.

The angular accelerations at the seat pan are measured by two SIT-pads whose sensitivities are $200 \mathrm{mV} / \mathrm{g}$ with the horizontal distance of $180 \mathrm{~mm}$. Each accelerometer is calibrated by giving zero reading when it is attached to a horizontal surface, and $+2 \mathrm{~g}$ when it is inversely placed. All the data is recorded at 512 samples per second via an antialiasing filter set at $40 \mathrm{~Hz}$.

2.3. Subjects. The 8 subjects participating in the current study are males: 25 to 42 years (30 years in average), 1.69 to $1.88 \mathrm{~m}$ in stature $(179.4 \mathrm{~cm}$ in average), and $65-95 \mathrm{~kg}$ in weight ( $75.6 \mathrm{~kg}$ in average). Informed consent is given by the subjects voluntarily. During the test, subjects are asked to sit on the rigid seat in an upright posture with no contact to the backrest and average thigh contact to the seat pan and a hand holding an emergency button throughout the test.

\section{Model Description}

3.1. Model with Roll Excitation. A two degrees-of-freedom (DOFs) model is used to represent the biodynamic response of human body exposed to random vibration in the roll direction (Figure 2). The two rigid bodies are used other than one to represent the banding motion of spine in lateral direction considering the layered structure of spine $[20,22,28]$. It is assumed that the sway and bending motion of spine can be achieved by rotational movements of a few rigid bodies without any translational DOFs. So, two linear torsional springs and dampers parallels, representing the interval discs between vertebras, are used to describe the connections between the upper body and the lower body and between the lower body and the basis. The rotational joints are set at the midpoint of two adjacent rigid bodies. The centre of gravity of each segment is defined in the geometric centre considering that the human body is symmetrical about the midsagittal plane. The geometric parameters such as the height of centres of gravity are obtained from Kitazaki [29]. The data are based on the median person. Hardly can inertia parameters be determined from literature or direct measurement. As a result, masses of the rigid bodies are estimated from Kitazaki's finite element model and moment of inertia is identified together with the stiffness and damping parameters later. The rotational motion of the upper body is $\theta_{2}$, the rotational motion of the lower body is $\theta_{1}$, and the input excitation is $\theta_{\mathrm{b}}$.

The equations of motion of the model can be derived by Lagrange equation:

$$
\frac{\mathrm{d}}{\mathrm{d} t}\left(\frac{\partial T}{\partial \dot{\mathbf{q}}}\right)+\frac{\partial D}{\partial \dot{\mathbf{q}}}+\frac{\partial U}{\partial \mathbf{q}}=0
$$

where $T$ is the kinetic energy, $D$ is the dissipating energy related to damping, $U$ is the potential energy, and $\boldsymbol{q}$ is $\boldsymbol{q}_{\boldsymbol{A}}=\left[\begin{array}{ll}\theta_{1} & \theta_{2}\end{array}\right]$. The equation of motion can be derived as

$$
\mathrm{J}_{\mathrm{A}} \ddot{\mathbf{q}}_{\mathrm{A}}+\mathrm{C}_{\mathrm{A}} \dot{\mathbf{q}}_{\mathrm{A}}+\mathbf{K}_{\mathrm{A}} \mathbf{q}_{\mathrm{A}}=\mathrm{T}_{\mathrm{A}}(\mathbf{t}),
$$

where $J_{\boldsymbol{A}}=\operatorname{diag} \quad\left(J_{1}, \quad J_{2}\right), \quad \mathrm{K}_{A}=\left(\begin{array}{cc}k_{1 A}+k_{2 A} & -k_{2 A} \\ -k_{2 A} & k_{2 A}\end{array}\right)$, $\mathbf{C}_{\mathbf{A}}=\left(\begin{array}{cc}c_{1 A}+c_{2 A} & -c_{2 A} \\ -c_{2 A} & c_{2 A}\end{array}\right)$, and $\mathbf{T}_{\mathbf{A}}(\mathbf{t})=\left(\begin{array}{c}k_{1 A} \theta_{b}+c_{1 A} \dot{\theta}_{b} \\ 0\end{array}\right)$.

3.2. Model with Lateral Excitation. To conduct comparisons between biodynamic responses especially the first mode of human body under roll and lateral vibration, another three DOFs model shown in Figure 3 is developed to characterise the roll motion of seated human body under lateral excitation. The models shown in Figures 2 and 3 share the same inertia and geometric parameters except that another DOF is added to the model in Figure 3 in the lateral direction. The stiffness and damping parameters are determined through the similar calibration procedure applied to roll model. The extra DOF, $x_{0}$, represents the translational displacement in the lateral direction of the whole body. The excitation is a translational displacement of seat pan at the buttocks tissue, $x_{\mathrm{b}}$.

The equations of the motion can be derived as

$$
\begin{aligned}
\mathbf{q}_{\mathbf{B}} & =\left[x_{0} \theta_{1} \theta_{2}\right], \\
T & =\frac{1}{2} \sum_{i=1}^{2} m_{i} \dot{x}_{i}^{2}+\frac{1}{2} \sum_{i=1}^{2} I_{i} \dot{\theta}_{i}^{2}, \\
U & =\frac{1}{2} k_{2 B}\left(\theta_{2 B}-\theta_{1 B}\right)^{2}+\frac{1}{2} k_{1 B} \theta_{1 B}^{2}+\frac{1}{2} k_{0}\left(x_{0}-x_{b}\right)^{2}, \\
D & =\frac{1}{2} c_{2 B}\left(\dot{\theta}_{2 B}-\dot{\theta}_{1 B}\right)^{2}+\frac{1}{2} c_{1 B} \dot{\theta}_{1 B}^{2}+\frac{1}{2} c_{0}\left(\dot{x}_{0}-\dot{x}_{b}\right)^{2} .
\end{aligned}
$$

The translational velocity of each segment is calculated as

$$
\begin{aligned}
& \dot{x}_{1}=\frac{1}{2} h_{1} \cdot \dot{\theta}_{1}+\dot{x}_{0}, \\
& \dot{x}_{2}=h_{1} \dot{\theta}_{1}+\frac{1}{2} h_{2} \dot{\theta}_{2}+\dot{x}_{0},
\end{aligned}
$$

where $x_{i}$ and $\dot{x}_{i}$ represent the translation displacement and velocity in the lateral direction of each segment in the model, $h_{i}$ refers to the height from the top of each rigid body to the rotational joint under it, and $m_{\mathrm{i}}$ and $J_{\mathrm{i}}$ are the mass and the moment of inertia of each part.

The equations of motion can be derived as

$$
\mathbf{M}_{\mathrm{B}} \ddot{\mathbf{q}}_{\mathrm{B}}+\mathrm{C}_{\mathrm{B}} \dot{\mathbf{q}}_{\mathrm{B}}+\mathrm{K}_{\mathrm{B}} \mathbf{q}_{\mathrm{B}}=\mathrm{T}_{\mathrm{B}}(\mathbf{t}),
$$




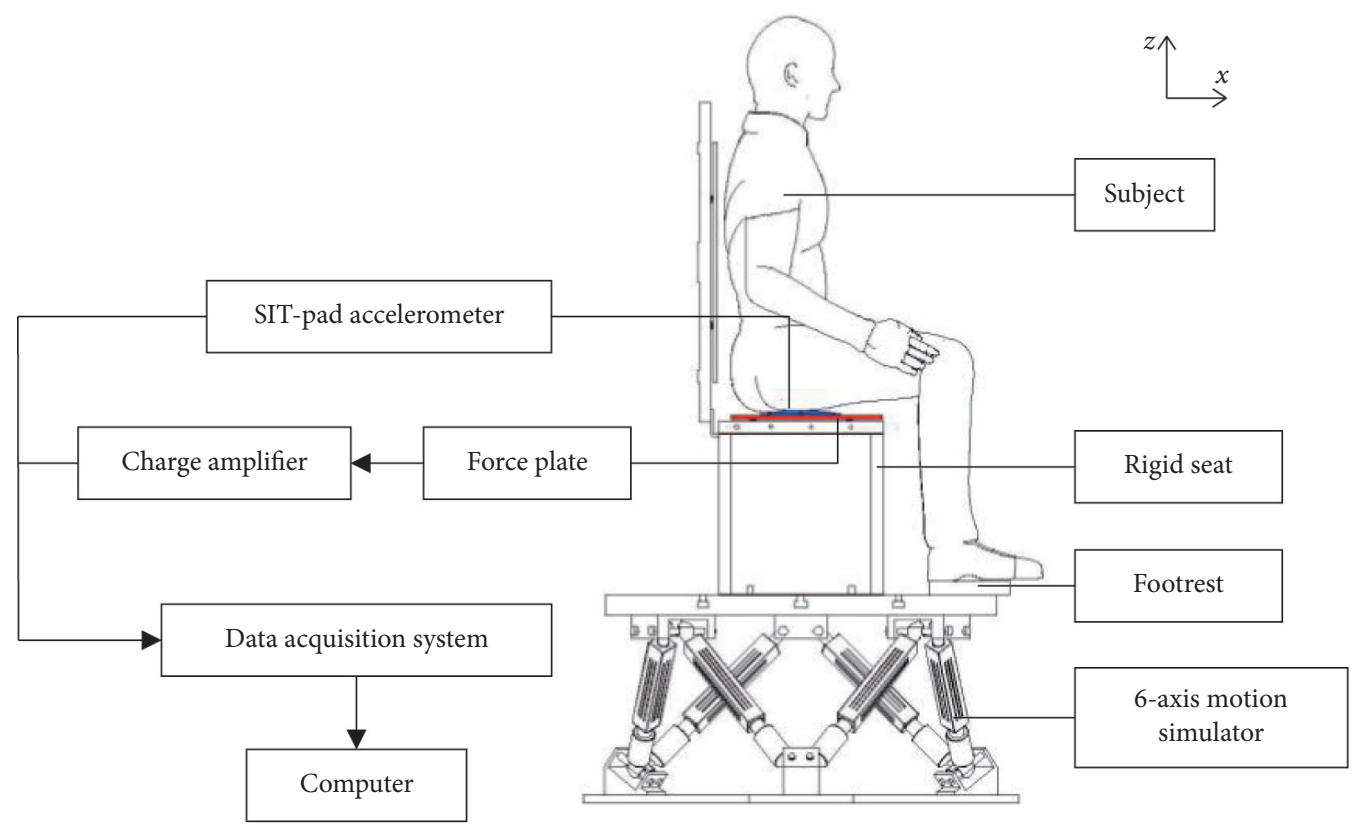

Figure 1: The experimental setup.

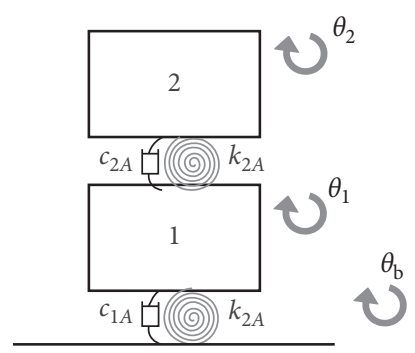

FIgURE 2: The roll response model with roll excitation.

$$
\begin{aligned}
& \left(\begin{array}{ccc}
m_{1}+m_{2} & 1 / 2 m_{1} h_{1}+m_{2} h_{1} & 1 / 2 m_{2} h_{2} \\
1 / 2 m_{1} h_{1}+m_{2} h_{1} & 1 / 4 h_{1}{ }^{2} m+m_{1} h_{1}^{2}+J_{1} & 1 / 2 m_{2} h_{2} h_{1} \\
1 / 2 m_{2} h_{2} & 1 / 2 m_{2} h_{2} h_{1} & 1 / 4 h_{2}^{2}+J_{2}
\end{array}\right) \\
& \mathbf{K}_{\mathbf{B}}=\left(\begin{array}{ccc}
k_{0} & 0 & 0 \\
0 & k_{1 B}+k_{2 B}-k_{2 B} \\
0 & -k_{2 B} & k_{2 B}
\end{array}\right), \quad \mathbf{C}_{\mathbf{B}}=\left(\begin{array}{ccc}
c_{0 B} & 0 & 0 \\
0 & c_{1 B}+c_{2 B}-c_{2 B} \\
0 & -c_{2 B} & c_{2 B}
\end{array}\right) \text { and } \\
& \mathbf{T}_{\mathbf{B}}(\mathbf{t})=\left(\begin{array}{c}
k_{0} x_{b}+c_{0} \dot{x}_{b} \\
0
\end{array}\right) \text {. }
\end{aligned}
$$

\section{Results and Discussion}

4.1. Apparent Inertia. Before the calculation of apparent inertia, mass cancellation for the moment of force at the seat pan is carried out by removing the moment of inertia of the aluminium plate above the force transducers. The moment of inertia of the aluminium, determined as the apparent inertia $8.7 \mathrm{kgm}^{2}$ at $1 \mathrm{~Hz}$ measured without subject, will be removed from the measurement with subjects. Apparent inertia of seated human body at four magnitudes of excitation is shown in Figure 4 and the median apparent inertia is shown in Figure 5.

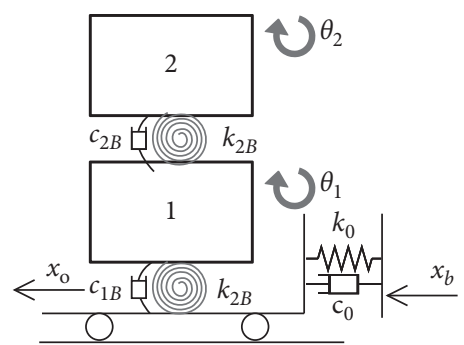

FIgURE 3: The roll response model with lateral excitation.

The principal resonance in the roll apparent inertia during random vibration is about $1 \mathrm{~Hz}$ but varied from 0.7 to $1.5 \mathrm{~Hz}$ depending on the magnitude of vibration $(0.5$ to $2.0 \mathrm{rad} / \mathrm{s}^{2}$ ) and subjects. Secondary resonance around $3 \mathrm{~Hz}$ can be found with much lower moduli and depends on the magnitude of excitation and changes between subjects (from $2.5 \mathrm{~Hz}$ to $3.25 \mathrm{~Hz}$ ). Considering that only two resonance peaks in apparent inertia are observed, it is reasonable to model the human body with two DOFs in Section 3.1. More DOFs in roll direction seem to have limited advantages to reflect the biodynamic response of seated human body. The transfer function of the roll response under roll excitation is similar to that of the roll response under lateral excitation reported in [10]. But the resonance frequencies $1 \mathrm{~Hz}$ for primary and $3 \mathrm{~Hz}$ for $\mathrm{sec}$ ondary under roll excitation are higher than the $0.7 \mathrm{~Hz}$ and $2 \mathrm{~Hz}$, respectively, under lateral excitation. The lower resonance frequencies, $0.7 \mathrm{~Hz}$ and $2 \mathrm{~Hz}$, may be accredited to the nonlinearity of human body. The random lateral excitation with $1.0 \mathrm{~m} / \mathrm{s}^{2}$ r.m.s. used in [10] can be equivalent to a $3.02 \mathrm{rad} / \mathrm{s}^{2}$ r.m.s. roll excitation approximately with the equivalent method introduced later in Section 4.6. Compared to the level of excitations, $0.5,1.0,1.5$, and $2.0 \mathrm{rad} / \mathrm{s}^{2}$ r.m.s. used in this study, it is reasonable that the 

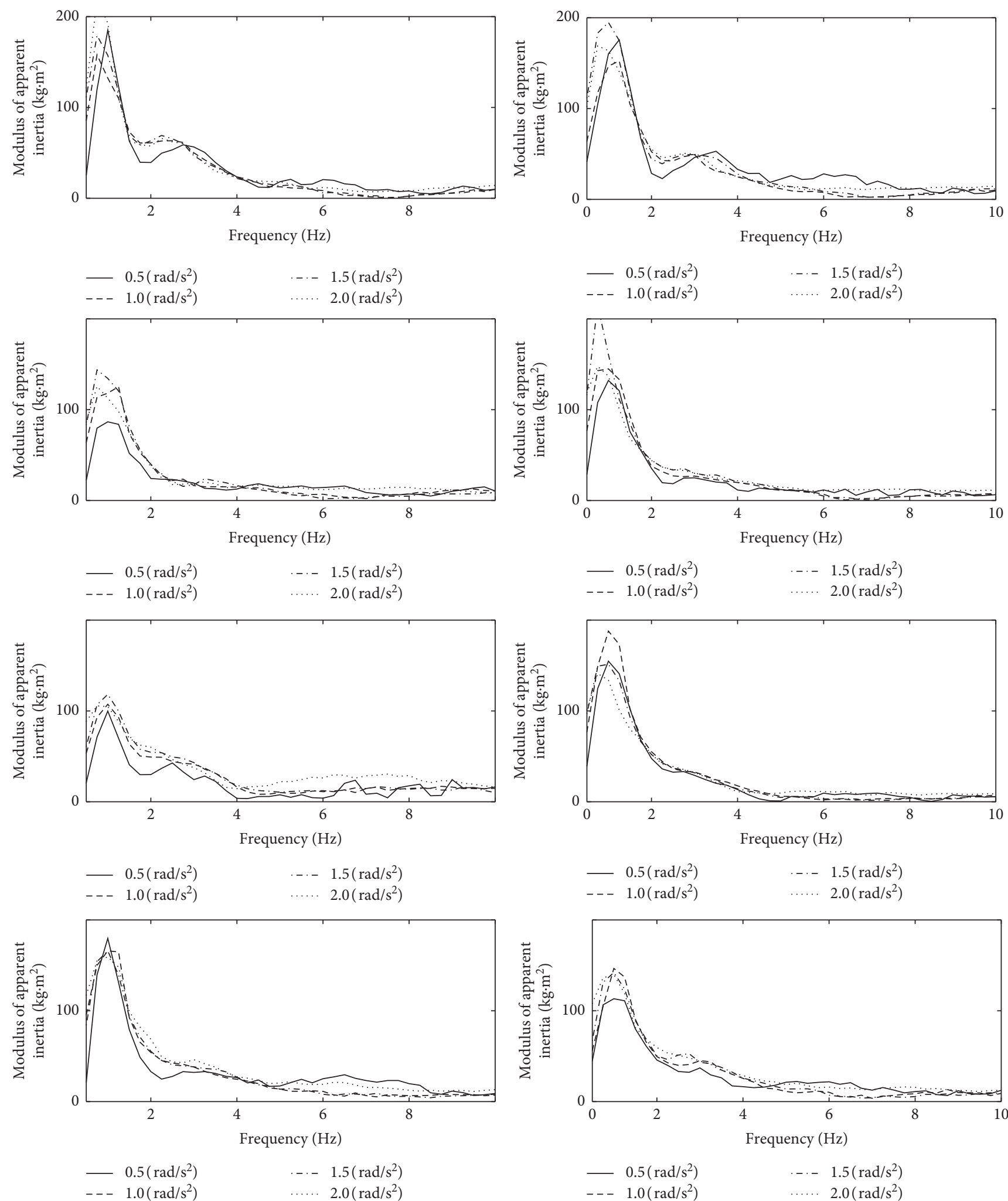

Figure 4: Apparent inertia of eight subjects at 4 magnitudes of excitation.

decrease of resonance frequency is observed with higher magnitude of excitation. There is another common point that the responses are slightly above $8 \mathrm{~Hz}$ for the roll motion under roll and lateral excitations.
The first mode is attributed to the motion of the whole upper body due to the sway-motion of the spine under roll excitation. The second mode appears to arise from the horizontal response of the muscular-skeletal structure of the 

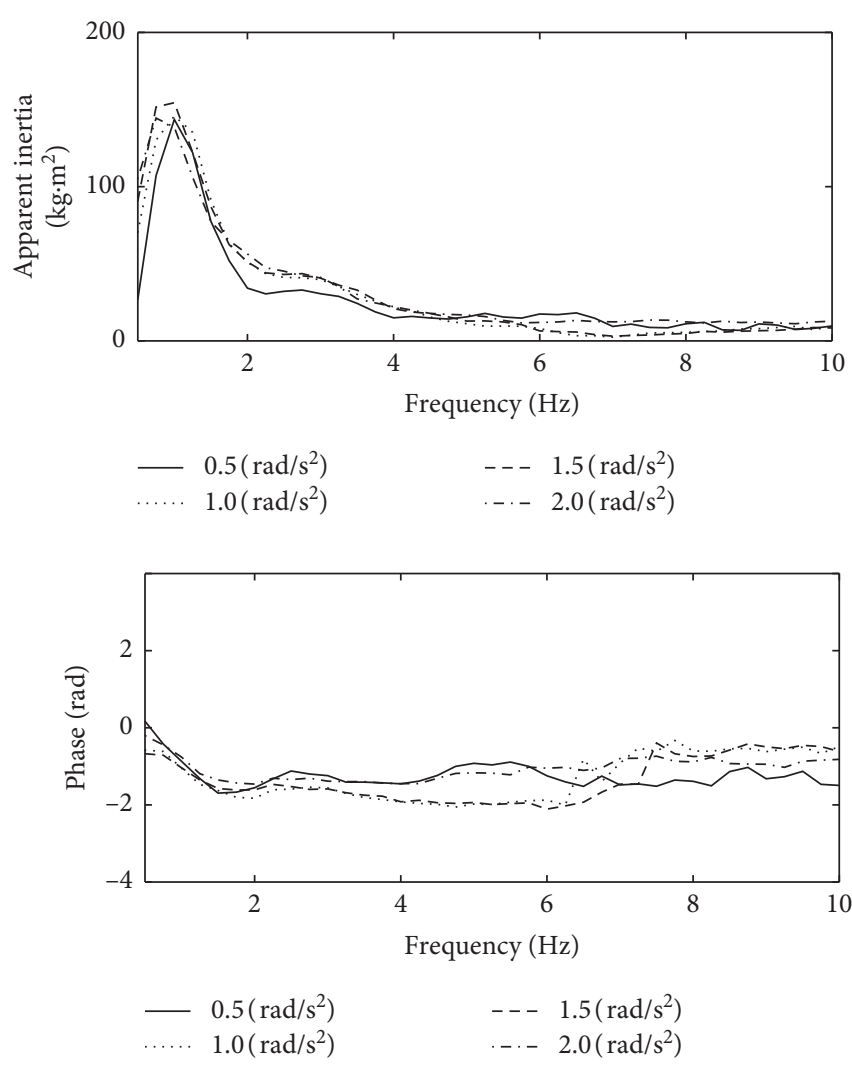

FigURe 5: Median apparent inertia of 8 subjects under roll vibration at 4 magnitudes.

torso: the motion of the ischium with its adjacent tissue is out of phase with the motion of shoulders.

4.2. Intersubject Variability. To remove the effect of the weight of subjects on the modulus of apparent inertia, the normalized apparent inertia which is shown in Figure 6 is calculated. The apparent inertia measured at $0 \mathrm{~Hz}$ is numerically equal to the partial static mass or moment of inertia of the subject seated on the force plate. Considering the frequency response limit of the transducers, the normalized apparent inertia is obtained by the ratio of apparent inertia at each frequency and that measured at $0.5 \mathrm{~Hz}$. The first resonance frequencies which vary from 0.75 to $1.25 \mathrm{~Hz}$ have little differences among subjects at a specific excitation while intersubject variability of the moduli of the normalized apparent inertia is observed. There was positive correlation between the mass of subjects and the modulus of the primary resonance. Differences of both the modulus and frequency of the second resonance are much more significant. It was noticed that the magnitude of apparent inertia on the second mode is associated with the weight of subjects. Subjects No. 1, No. 2, and No. 8 who are the three heaviest subjects in the test had more pronounced responses on the second mode, which implies the second resonance observed in the experiment is not determined by the motion of the spine but associated with muscle and fat. The average coefficients of variation, the average of the ratios of the standard deviations to the means calculated at each frequency [11], at four vibration magnitudes are $0.33,0.27,0.23$, and 0.22 , which indicates that higher variability occurred at lower excitation magnitude.

4.3. Comparison with Seat-to-Head Transmissibility. The seat-to-head transmissibility of human body under roll vibration was obtained by Paddan and Griffin [27]. Compared with the apparent inertia measured in the study, similar primary resonance frequency is observed, while the secondary mode cannot be detected. The absence of the second resonance in the seat-to-head transmissibility is consistent with the analysis that the second mode of human body under roll vibration is not related to the motion of whole upper body dominated by the spine but muscle and fat of the torso. Another difference between seat-to-head transmissibility and apparent inertia is about the intersubject variability. The intersubject variability of apparent inertia is not as conspicuous as that of the transmissibility. It had been shown that the sitting posture which was not easy to be controlled during the experiment was the most likely to affect the transmissibility [30]. As a result, apparent inertia is better for the statistical analysis to obtain the biodynamic characteristics of human body under roll vibration.

4.4. Effects of Magnitude on Apparent Inertia. The effect of magnitude on apparent inertia is analysed with the median of apparent inertia of 8 subjects, shown in Figure 5. Statistical analysis is conducted, which showed there is no significant change of the first resonance frequency with increased magnitude of excitation. Nonlinearity is expected but not observed at the first mode, which may result from the subject's involuntarily control in the lower back to restrain the rocking in case of falling from the seat. In contrast, the nonlinear behaviour on the second mode will not be influenced. Similar nonlinear behaviour was reported by Fairley [10] in an experimental study to the fore-and-aft and lateral apparent inertia of seated human body. The second peak frequency decreases when the magnitude of excitation increases especially from $0.5 \mathrm{rad} / \mathrm{s}^{2}$ to $1 \mathrm{rad} / \mathrm{s}^{2}$. The nonlinearity of the second mode is more pronounced than that of the first mode. Besides, statistical analysis also showed evident increase of moduli of the first resonance with increased excitation.

4.5. Model Stiffness and Damping. To identify the parameters in models $\mathrm{A}$ and $\mathrm{B}$, the error function is defined as

$$
E(\lambda)=\sqrt{\frac{1}{N} \sum_{i=1}^{N}\left(\left|M_{p_{-} \text {mod }}\left(f_{i}\right)\right|-\left|M_{m_{\text {_mod }}}\left(f_{i}\right)\right|\right)^{2}+\frac{1}{N} \sum_{i=1}^{N}\left(\left|M_{p_{\text {_ang }}}\left(f_{i}\right)\right|-\left|M_{m_{-} \text {ang }}\left(f_{i}\right)\right|\right)^{2}},
$$



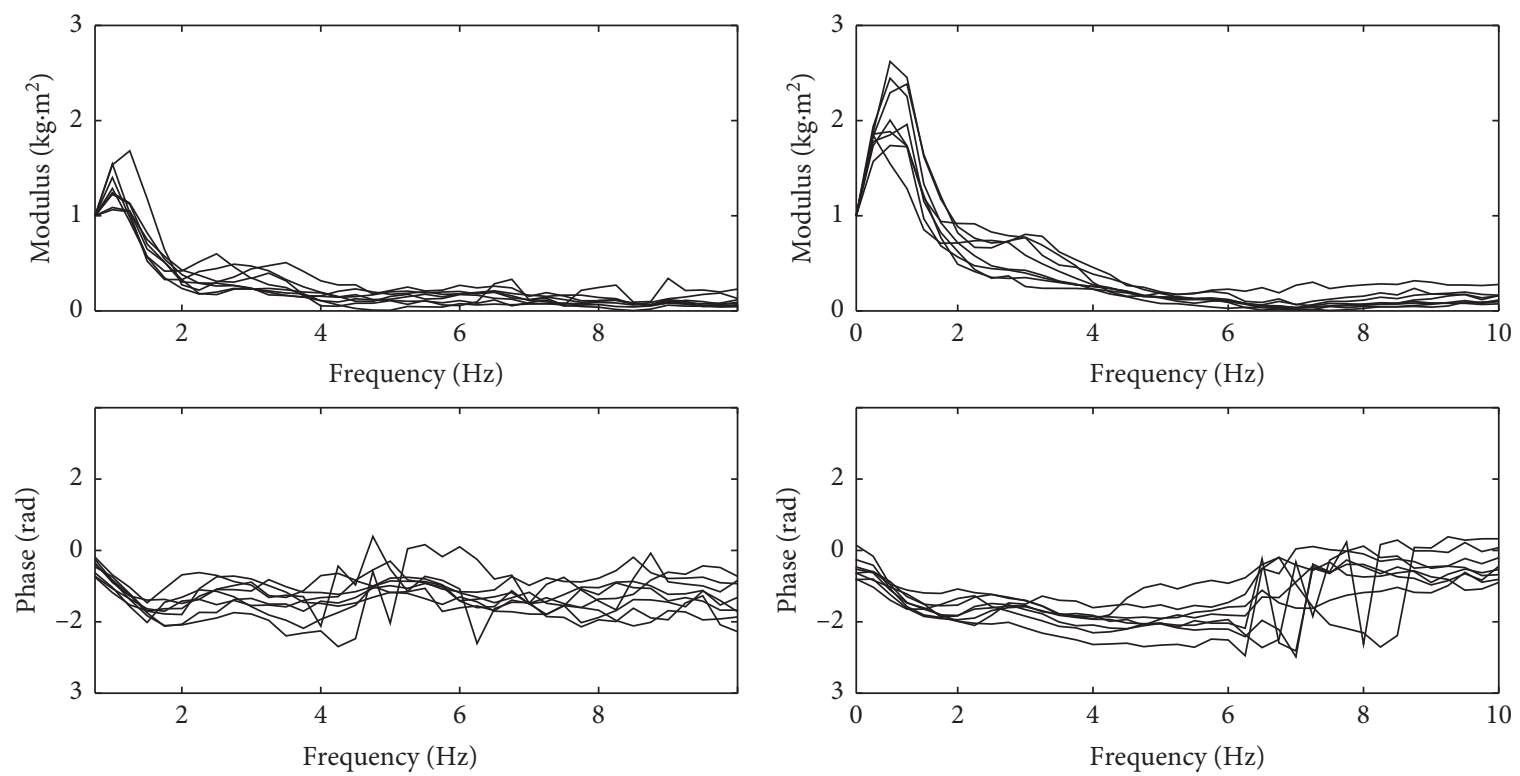

(a)

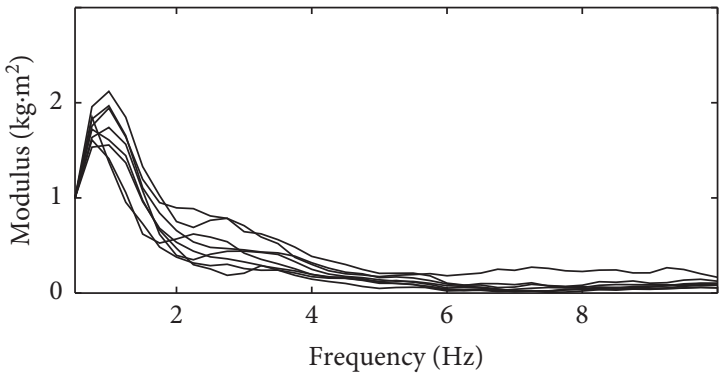

(b)

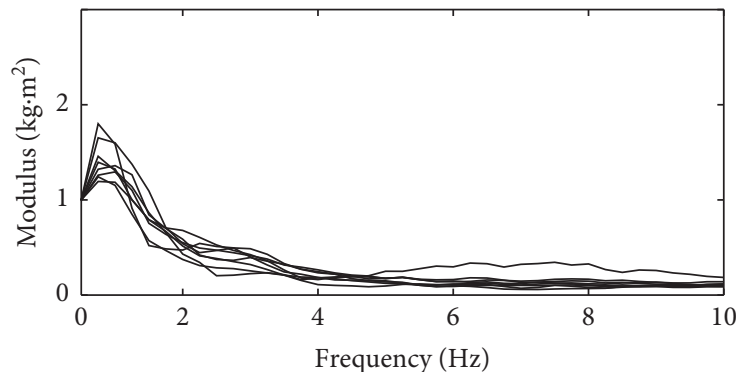

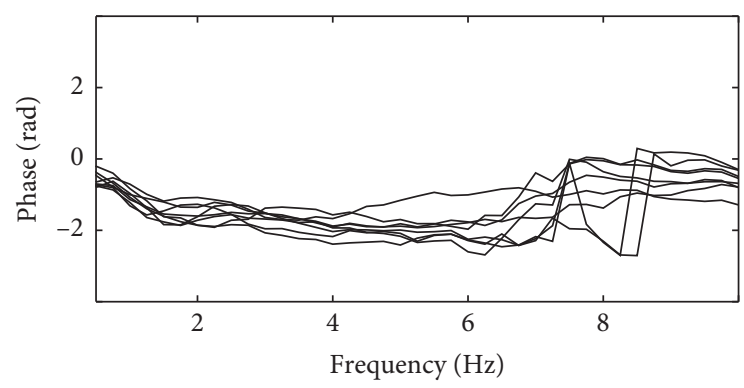

(c)

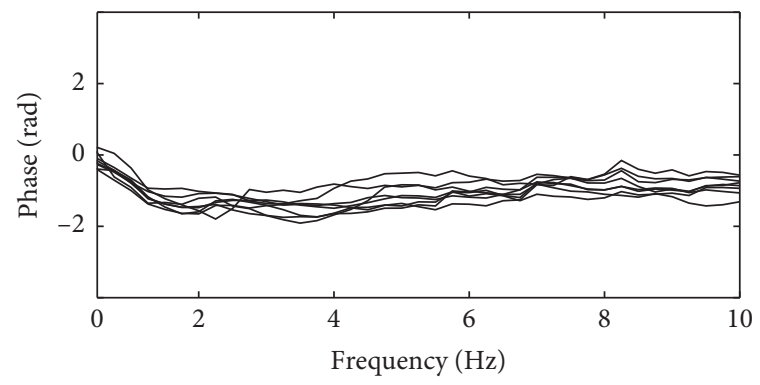

(d)

FiguRe 6: Intersubject variability: normalized apparent inertia of eight subjects at 4 magnitudes of excitation: (a) $0.5 \mathrm{rad} / \mathrm{s}^{2} \mathrm{r} . \mathrm{m} . \mathrm{s} . ;$ (b) $1.0 \mathrm{rad} / \mathrm{s}^{2}$ r.m.s.; (c) $1.0 \mathrm{rad} / \mathrm{s}^{2}$ r.m.s.; (d) $1.0 \mathrm{rad} / \mathrm{s}^{2}$ r.m.s.

where $M_{\text {p_mod }}\left(f_{i}\right)$ and $M_{m_{-} \text {ang }}\left(f_{i}\right)$ are the modulus and phases predicted by models proposed while $M_{m_{-} \bmod }\left(f_{i}\right)$ and $M_{m_{-} \text {ang }}\left(f_{i}\right)$ are the modulus and phase measured in the experiment carried out in Section 2 and by Fairley [10]. The error is a function of $\lambda$ containing model parameters. With $\lambda_{\mathrm{A}}=\left(J_{1} J_{2} k_{1 A} c_{1 A} k_{2 A} c_{2 A}\right)^{T}$ and $\lambda_{\mathbf{B}}=\left(k_{1 B} c_{1 B} k_{2 B} c_{2 B}\right)^{T}$, two models were calibrated by searching the minimum value of error function over the frequency range 0.5 to $10 \mathrm{~Hz}$. The model parameters are listed in Table 1.

As shown in Figure 7, the modulus of the roll and lateral apparent inertia predicted by the models are in close agreement with test results. The primary peak of roll apparent inertia predicted by model shows similar frequency and modulus with measured data while the resonance around $3 \mathrm{~Hz}$ cannot be obtained attributed to the absence of the lateral DOF which reflects the horizontal motion of muscular-skeletal structure of the upper body.

4.6. Modal Analysis. Modal analysis with the models is carried out after they are calibrated, which benefits understanding of the connection between biodynamic characteristics of human body under roll and lateral vibration. It is found that the modal frequencies are related to the resonances in the measured apparent inertia both in lateral and 
TABLE 1: Inertia, stiffness, and damping parameters used in the dynamic models.

\begin{tabular}{|c|c|c|c|c|c|}
\hline \multicolumn{2}{|c|}{ Inertia } & \multicolumn{2}{|c|}{ Stiffness } & \multicolumn{2}{|c|}{ Damping } \\
\hline$J_{1}$ & $55.2 \mathrm{~kg} \cdot \mathrm{m}^{2}$ & $k_{1 A}$ & $6.47 \times 10^{3} \mathrm{Nm} / \mathrm{rad}$ & $c_{1 A}$ & $450 \mathrm{Nms} / \mathrm{rad}$ \\
\hline$J_{2}$ & $23.1 \mathrm{~kg} \cdot \mathrm{m}^{2}$ & $k_{2 A}$ & $1.98 \times 10^{3} \mathrm{Nm} / \mathrm{rad}$ & $c_{2 A}$ & $141 \mathrm{Nms} / \mathrm{rad}$ \\
\hline$M_{1}$ & $25.0 \mathrm{~kg}$ & $k_{1 B}$ & $2.36 \times 10^{3} \mathrm{Nm} / \mathrm{rad}$ & $c_{1 B}$ & $372 \mathrm{Nms} / \mathrm{rad}$ \\
\hline$M_{2}$ & $42.0 \mathrm{~kg}$ & $k_{2 B}$ & $1.86 \times 10^{3} \mathrm{Nm} / \mathrm{rad}$ & $c_{2 B}$ & $138 \mathrm{Nms} / \mathrm{rad}$ \\
\hline & & $k_{0}$ & $2.50 \times 10^{3} \mathrm{~N} / \mathrm{m}$ & $c_{0}$ & $264 \mathrm{Ns} / \mathrm{m}$ \\
\hline
\end{tabular}

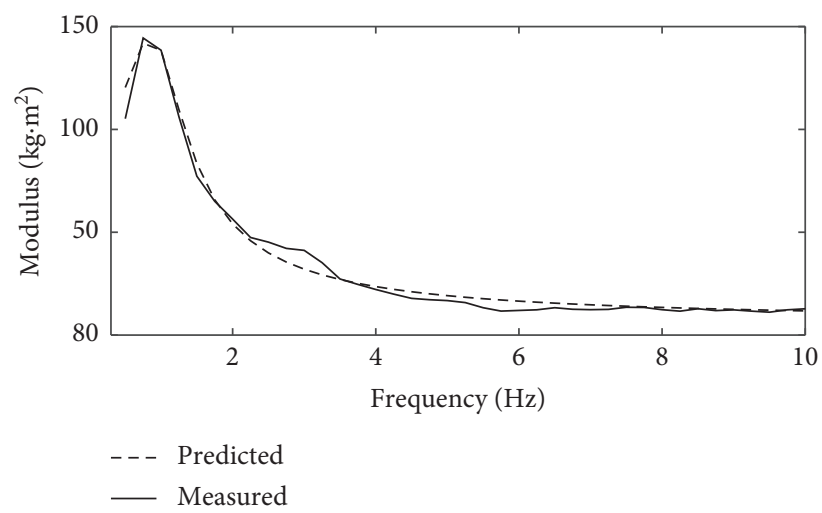

(a)

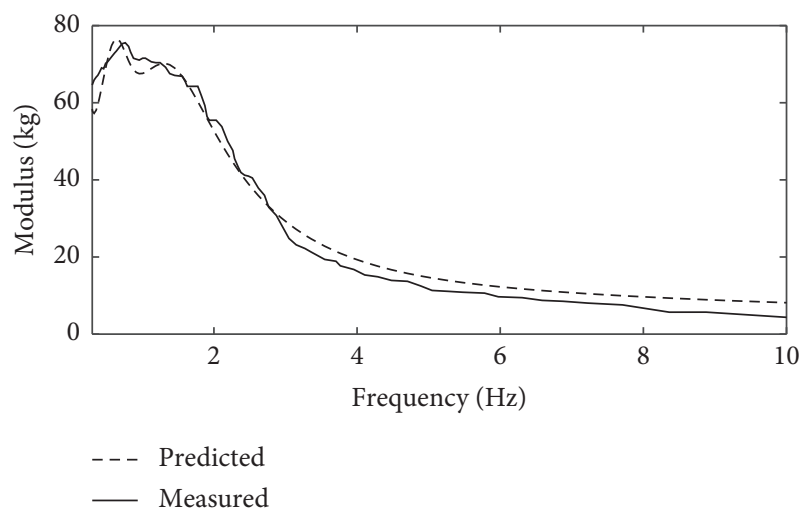

(b)

FIgURe 7: Measured and predicted apparent inertia by the model under. (a) Roll vibration and (b) lateral vibration.

in roll direction. The first mode at about $0.7 \mathrm{~Hz}$ is detected in both models. The corresponding mode shapes of two models are $(0.36601 .000)^{T}$ for model A and $(0.062200 .1120$ $1.000)^{T}$ for model B. The mode shapes illustrate that the first mode of human body under roll and lateral excitation are both dominated by the rocking and sway of the whole upper body instead of bending motion of the spine which determined the second mode with shapes $(-1.1411 .000)^{T}$ and $(-0.6237-0.69851 .000)^{T}$ for roll model and lateral model. The primary and secondary mode shapes are shown in Figure 8. The results imply the possibility that the movement patterns at the first resonance frequency of human body exposed to roll and lateral vibration are similar, which makes it reasonable to conduct more detailed analysis and establish the connection between the human response under roll and lateral vibration to benefit the study of biodynamic characteristics of human body under multiaxis excitation.

4.7. Correlation between Roll and Lateral Vibration. According to above results, it is worthy to analyse the correlation between the responses of seated human body when exposed to roll and lateral vibrations. The lateral apparent inertia obtained by Fairley [10] was used to conduct the comparison with the roll data obtained in this study. Nondimensionalization was carried out considering that the modulus of lateral apparent mass and the roll apparent inertia were incomparable with different units. The nondimensionalized transfer functions of seated human body were obtained by the ratio of apparent mass or apparent inertia at each frequency and that measured at $0.25 \mathrm{~Hz}$, which are shown in Figure 9.
It is observed that the nondimensionalized roll and lateral apparent inertia shared similar primary resonance frequency and modulus (roll: 1.373 at $0.75 \mathrm{~Hz}$; lateral: 1.355 at $0.70 \mathrm{~Hz}$ ). Considering the effects of nonlinearity and the magnitude of excitation, the similarity between the first resonance frequency of lateral and roll apparent inertia is considerable. In contrast, for the secondary resonance, both the frequency and amplitude are conspicuously different, which implies the motion determined by the second mode of roll apparent inertia differs from that of lateral apparent inertia. The second resonance of upright seated human body under lateral excitation may be dominated by the bend of spine, which is different from that under roll excitation: the motion of muscular-skeleton structure.

The linear correlation analysis is conducted. It can be assumed that the measured horizontal force over the interface of human body and seat pan excited by lateral vibration is the superposition of the consequence of the lateral movement and rotation of the upper body. The Pearson correlation coefficient calculated with nondimensionalised lateral apparent mass and roll apparent inertia is 0.972 . Since the linear correlation between the lateral and roll response is strong enough-i.e., the Pearson correlation coefficient is close to the unity-it can be concluded that the lateral response excited by lateral vibration accounted for a small proportion of the overall response. Instead, the roll response that can also be excited by roll excitation dominates the motion of human body even if it is exposed to lateral vibration. Since the two kinds of excitation share similar responses in roll direction, it can be deduced that the scale factor between responses under roll and lateral excitations was related to the square of the arm of measured lateral force. 


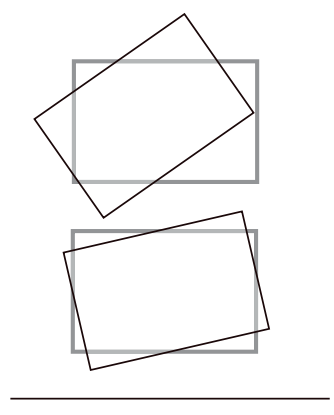

(a)

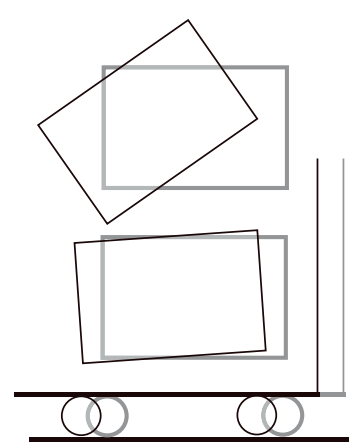

(b)

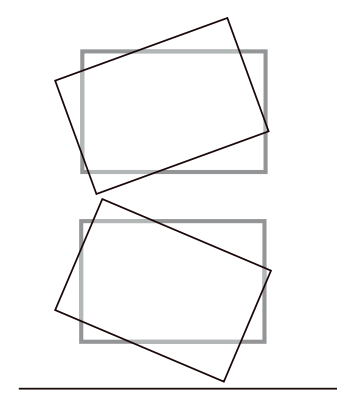

(c)

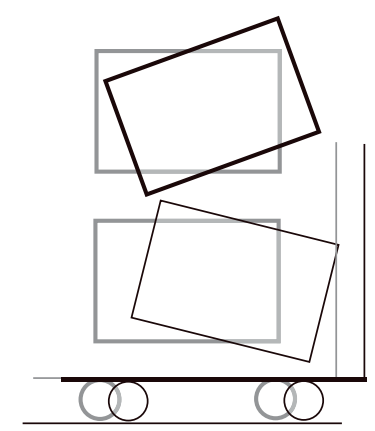

(d)

Figure 8: Mode shape of the roll and lateral model corresponding to the primary and secondary mode. (a) Roll model for primary mode; (b) lateral model for primary mode; (c) roll model for secondary mode; (d) lateral model for secondary mode.

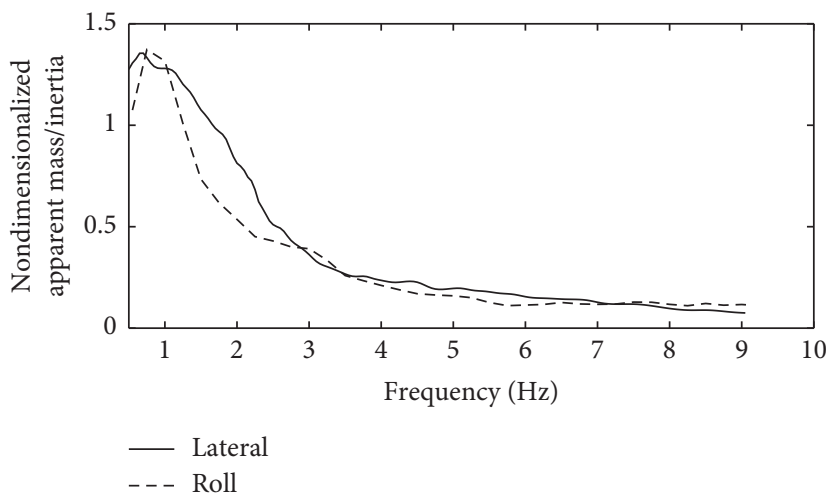

Figure 9: Nondimensionalized apparent inertia of seated human body under roll and lateral vibrations.

It is reasonable to establish the equivalent relationship between roll and lateral responses by the models developed in Section 3. The biodynamic responses of rigid body \#2 of model A and model B under simple-harmonic excitation can be calculated in the frequency domain with

$$
\begin{aligned}
& Y_{2 A}\left(f, E_{R}\right)=H_{\theta_{2} \theta_{b}}(f) \times \Theta\left(f, E_{R}\right), \\
& Y_{2 B}\left(f, E_{L}\right)=H_{\theta_{2} x_{b}}(f) \times X\left(f, E_{L}\right),
\end{aligned}
$$

where $\Theta\left(f, E_{R}\right)$ and $X\left(f, E_{L}\right)$ are the roll and lateral excitations which are functions of frequency $(f)$ as well as magnitude and phase of the simple-harmonic excitations acting on the models. $E_{R}$ and $E_{L}$ represent the roll and lateral simple-harmonic excitations in a complex number, respectively. $H_{\theta_{2} \theta_{b}}(f)$ and $H_{\theta_{2} x_{b}}(f)$ are the transfer functions from roll excitation and lateral excitation to the rotation responses of rigid body \#2, $Y_{2 A}$ and $Y_{2 B}$. To figure out the equivalent magnitudes of roll and lateral excitation that cause identical response of the upper human body, let $Y_{2 A}$ and $Y_{2 B}$ be equal; then the relationship between roll and equivalent lateral excitation can be derived as

$$
\Theta\left(f, E_{R}\right)=\frac{H_{\theta_{2} x_{b}}(f)}{H_{\theta_{2} \theta_{b}}(f)} X\left(f, E_{L}\right) .
$$

The magnitude and phase of roll excitation and equivalent lateral excitation vary with frequency; see Figure 10.
Each point on the equivalence surface has the same rotational response of upper human body under corresponding roll and lateral excitations. It can be concluded that, at lower frequency (below $3 \mathrm{~Hz}$ ), roll excitation, whose rotational centre is located on the midsagittal plane of human body, will cause more pronounced human response of upper body than lateral excitation with numerically identical amplitude. In contrast, at higher frequency the lateral vibration with the same level will produce greater response than roll vibration.

The equivalence method of roll and lateral excitations is applied to obtain equivalent roll and lateral vibration. It can be found that roll and lateral vibrations which share numerically identical amplitude, $1.4 \mathrm{~m} / \mathrm{s}^{2}$ r.m.s. and $1.4 \mathrm{rad} / \mathrm{s}^{2}$ r.m.s., with approximately flat constant bandwidth acceleration power spectrum defined in $0.5-10 \mathrm{~Hz}$ will not produce identical response of upper body. The power spectrum densities (PSDs) of angular acceleration of rigid body \#2 in roll and lateral models are calculated. The magnitudes of PSDs at the primary resonance frequency are quite different from each other, which is shown in Figure 11. Through (10), the roll vibration at magnitude of $4.7 \mathrm{rad} / \mathrm{s}^{2}$ r.m.s., which is equivalent to the $1.4 \mathrm{~m} / \mathrm{s}^{2}$ r.m.s. lateral excitation, is obtained. The equivalent procedure includes three steps: (a) FFT of the original $1.4 \mathrm{~m} / \mathrm{s}^{2}$ r.m.s. lateral excitation signal, (b) equivalence of the signal through equation (10) carried out in every frequency, and (c) transformation from the frequency domain to the time 


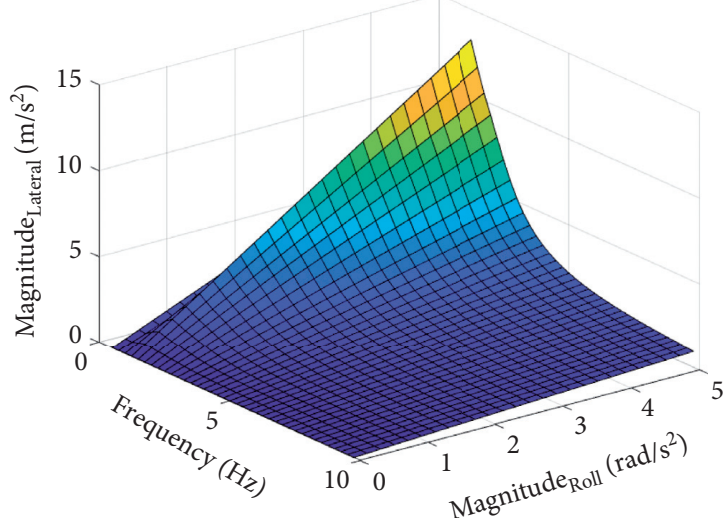

(a)

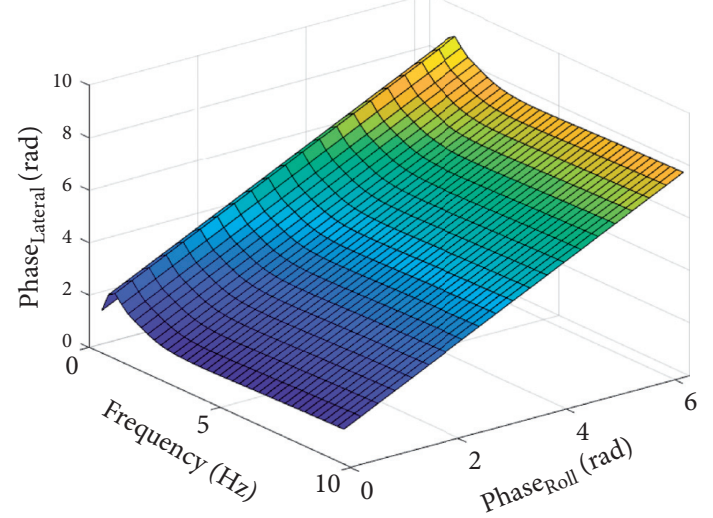

(b)

FIGURE 10: Equivalent surface of roll and lateral excitation in (a) magnitude and (b) phase.

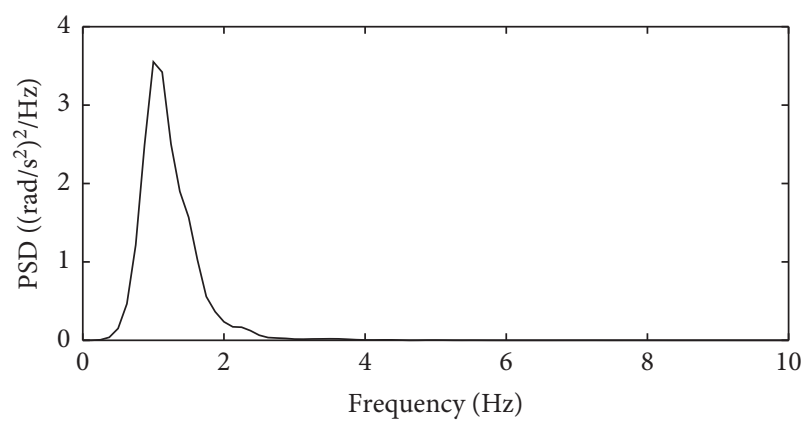

(a)

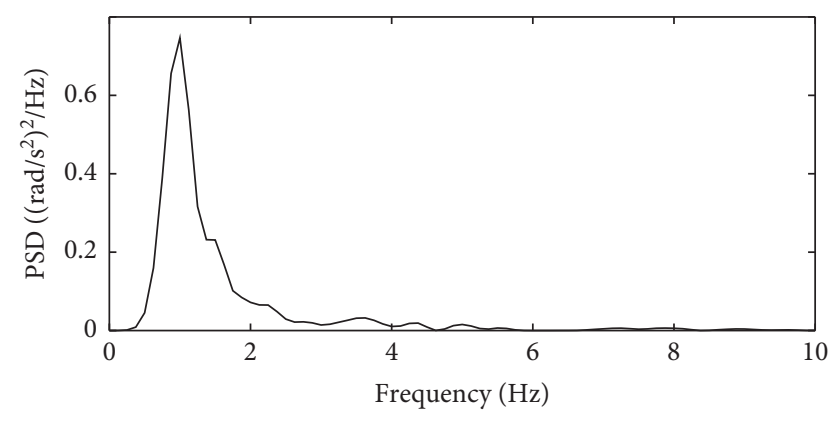

(b)

FIGURE 11: Roll response of upper human body under. (a) $1.4 \mathrm{rad} / \mathrm{s}^{2} \mathrm{r} . \mathrm{m} . \mathrm{s}$. roll vibration and (b) $1.4 \mathrm{~m} / \mathrm{s}^{2} \mathrm{r} . \mathrm{m} . \mathrm{s}$. lateral excitation.

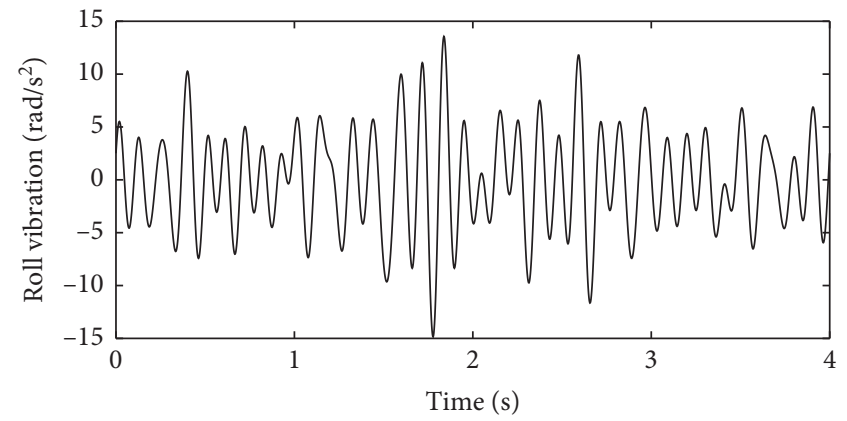

(a)

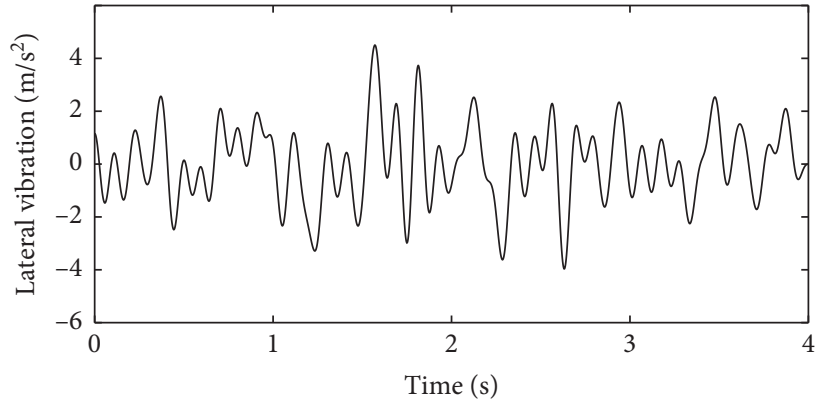

(b)

Figure 12: Equivalent $7.4 \mathrm{rad} / \mathrm{s}^{2}$ r.m.s. roll excitation and $1.4 \mathrm{~m} / \mathrm{s}^{2}$ r.m.s. lateral excitation.

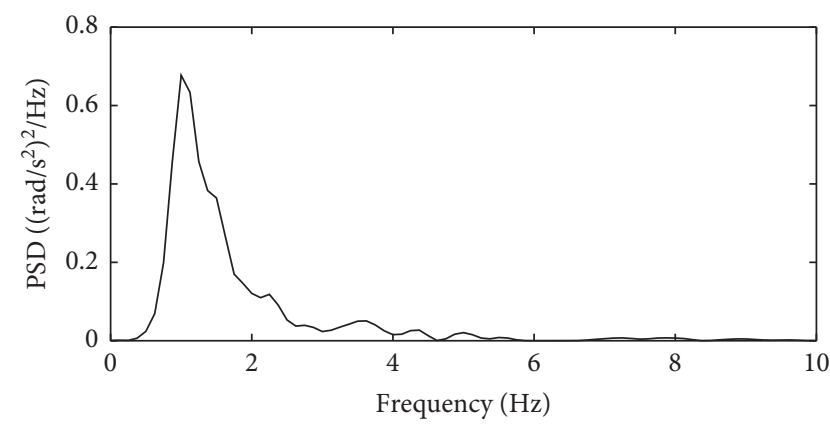

(a)

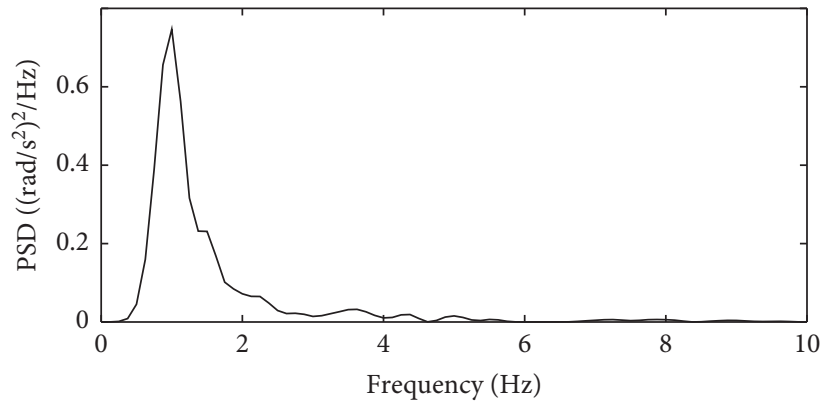

(b)

FIGURE 13: Roll response of upper human body under. (a) $4.7 \mathrm{rad} / \mathrm{s}^{2} \mathrm{r} . \mathrm{m} . \mathrm{s}$. roll vibration (equivalent with $1.4 \mathrm{~m} / \mathrm{s}^{2} \mathrm{r} . \mathrm{m} . \mathrm{s}$. lateral excitation) and (b) $1.4 \mathrm{~m} / \mathrm{s}^{2}$ r.m.s. lateral excitation. 
domain. The equivalent roll and lateral signals are shown in Figure 12. The PSDs of dynamic responses of the upper body are calculated by the roll model under $4.7 \mathrm{rad} / \mathrm{s}^{2} \mathrm{r}$.m.s. roll excitation and the lateral model under $1.4 \mathrm{~m} / \mathrm{s}^{2}$ r.m.s. lateral excitation, which are shown in Figure 13. It is observed that the upper body response under two different excitations shows similar responses, PSDs.

\section{Conclusion}

Under roll excitation, the apparent inertia shows a primary resonance around $1 \mathrm{~Hz}$ and a secondary peak about $3 \mathrm{~Hz}$. The primary resonant vibration is dominated by the sway motion of the whole upper body and the second one is attributed to the motion of muscular-skeletal structure around shoulders.

Inter-ubject variability is observed in the test and it seems to decrease with increasing vibration magnitude. The nonlinearity of human body under roll vibration is not pronounced.

Strong linear correlation is found between biodynamic responses of seated human body under roll and lateral vibrations, which indicates the roll motion is the main component of dual-axial movements excited by lateral vibration.

Equivalent relationship of roll and lateral excitations is developed under the condition of causing similar rotation responses of the upper body. A case study that converts a lateral excitation to an equivalent roll vibration shows the availability of the proposed equivalence approach.

\section{Data Availability}

The data used to support the findings of this study are available from the corresponding author upon request.

\section{Conflicts of Interest}

The authors declare that they have no conflicts of interest.

\section{Acknowledgments}

The authors gratefully acknowledge the project supported by the National Natural Science Foundation of China (51705357) and the project supported by Natural Science Foundation of Tianjin (China) (18JCYBJC20000).

\section{References}

[1] P. Manfred, Road and Off-Road Vehicle System Dynamics Handbook, CRC Press, 2018.

[2] X. Wu, S. Rakheja, and P.-É. Boileau, "Analyses of relationships between biodynamic response functions," Journal of Sound and Vibration, vol. 226, no. 3, pp. 595-606, 1999.

[3] T. E. Fairley and M. J. Griffin, "The apparent mass of the seated human body: vertical vibration," Journal of Biomechanics, vol. 22, no. 2, pp. 81-94, 1989.

[4] Y. Qiu and M. J. Griffin, "Biodynamic responses of the seated human body to single-axis and dual-axis vibration," Industrial Health, vol. 48, no. 5, pp. 615-627, 2010.
[5] V. Kumar and V. H. Saran, "Biodynamic model of the seated human body under the vertical whole body vibration exposure," International Journal of Acoustics \& Vibration, vol. 24, no. 4, 2019.

[6] N. J. Mansfield and M. J. Griffin, "Non-linearities in apparent mass and transmissibility during exposure to whole-body vertical vibration," Journal of Biomechanics, vol. 33, no. 8, pp. 933-941, 2000.

[7] Y. Matsumoto and M. J. Griffin, "Non-linear characteristics in the dynamic responses of seated subjects exposed to vertical whole-body vibration," Journal of Biomechanical Engineering, vol. 124, no. 5, pp. 527-532, 2002.

[8] N. Nawayseh and M. J. Griffin, "Non-linear dual-axis biodynamic response to vertical whole-body vibration," Journal of Sound and Vibration, vol. 268, no. 3, pp. 503-523, 2003.

[9] Y. Huang and M. J. Griffin, "Nonlinear dual-axis biodynamic response of the semi-supine human body during vertical whole-body vibration," Journal of Sound and Vibration, vol. 312, no. 1-2, pp. 296-315, 2008.

[10] T. E. Fairley and M. J. Griffin, "The apparent mass of the seated human body in the fore-and-aft and lateral directions," Journal of Sound and Vibration, vol. 139, no. 2, pp. 299-306, 1990.

[11] N. Nawayseh and M. J. Griffin, "Tri-axial forces at the seat and backrest during whole-body fore-and-aft vibration," Journal of Sound and Vibration, vol. 281, no. 3-5, pp. 921-942, 2005.

[12] N. Nawayseh, S. Alteneiji, and S. Hamdan, "Effect of gender on the biodynamic responses to vibration induced by a wholebody vibration training machine," Proceedings of the Institution of Mechanical Engineers, Part H: Journal of Engineering in Medicine, vol. 233, no. 3, pp. 383-392, 2019.

[13] N. Sinan and S. Hamdan, "Apparent mass of the standing human body when using a whole-body vibration training machine: effect of knee angle and input frequency," Journal of Biomechanics, vol. 82, pp. 291-298, 2019.

[14] Z. Gan, A. J. Hillis, and J. Darling, "Biodynamic modelling of seated human subjects exposed to uncouples vertical and fore-and-aft whole-body vibration," Journal of Vibration Engineering and Technologies, vol. 3, no. 3, pp. 301-314, 2015.

[15] N. J. Mansfield and S. Maeda, "Comparison of the apparent masses and cross-axis apparent masses of seated humans exposed to single- and dual-axis whole-body vibration," Journal of Sound and Vibration, vol. 298, no. 3, pp. 841-853, 2006.

[16] Y. Qiu and M. J. Griffin, "Biodynamic response of the seated human body to single-axis and dual-axis vibration: effect of backrest and non-linearity," Industrial Health, vol. 50, no. 1, pp. 37-51, 2012.

[17] G. Zheng, Y. Qiu, and M. J. Griffin, "Fore-and-aft and dualaxis vibration of the seated human body: nonlinearity, crossaxis coupling, and associations between resonances in the transmissibility and apparent mass," International Journal of Industrial Ergonomics, vol. 69, pp. 58-65, 2019.

[18] A. Błażejewski, S. Głowiński, and I. Maciejewski, “The wavelet transfer function of a human body-seat system," Journal of Low Frequency Noise, Vibration and Active Control, vol. 38, no. 2, pp. 817-825, 2019.

[19] Y. Matsumoto and M. J. Griffin, "Movement of the upperbody of seated subjects exposed to vertical whole-body vibration at the principal resonance frequency," Journal of Sound and Vibration, vol. 215, no. 4, pp. 743-762, 1998.

[20] Y. Matsumoto and M. J. Griffin, "Modelling the dynamic mechanisms associated with the principal resonance of the seated human body," Clinical Biomechanics, vol. 16, pp. S31-S44, 2001. 
[21] N. Nawayseh and M. J. Griffin, "A model of the vertical apparent mass and the fore-and-aft cross-axis apparent mass of the human body during vertical whole-body vibration," Journal of Sound and Vibration, vol. 319, no. 1-2, pp. 719-730, 2009.

[22] M. Yang, "Effects of sitting posture and seat backrest on the biodynamic response of the human body and the prediction of spinal forces during vertical whole-body vibration," in Faculty of Engineering and the EnvironmentUniversity of Southampton, Southampton, UK, 2016.

[23] K. C. Parsons and M. J. Griffin, "The effect of the position of the axis of rotation on the discomfort caused by whole-body roll and pitch vibrations of seated persons," Journal of Sound and Vibration, vol. 58, no. 1, pp. 127-141, 1978.

[24] G. F. Beard and M. J. Griffin, "Discomfort caused by low-frequency lateral oscillation, roll oscillation and roll-compensated lateral oscillation,” Ergonomics, vol. 56, no. 1, pp. 103-114, 2013.

[25] G. F. Beard and M. J. Griffin, "Discomfort of seated persons exposed to low frequency lateral and roll oscillation: effect of seat cushion," Applied Ergonomics, vol. 45, no. 6, pp. 1547-1557, 2014.

[26] G. F. Beard and M. J. Griffin, "Discomfort of seated persons exposed to low frequency lateral and roll oscillation: effect of backrest height," Applied Ergonomics, vol. 54, pp. 51-61, 2016.

[27] G. S. Paddan and M. J. Griffin, "Transmission of roll and pitch seat vibration to the head," Ergonomics, vol. 37, no. 9, pp. 1513-1531, 1994.

[28] S. Pankoke, B. Buck, and H. P. Woelfel, "Dynamic FE model of sitting man adjustable to body height, body mass and posture used for calculating internal forces in the lumbar vertebral disks," Journal of Sound and Vibration, vol. 215, no. 4, pp. 827-839, 1998.

[29] S. Kitazaki and M. J. Griffin, "Resonance behaviour of the seated human body and effects of posture," Journal of Biomechanics, vol. 31, no. 2, pp. 143-149, 1997.

[30] A. Messenger and M. J. Griffin, Effects of Anthropometric and Postural Variables on the Transmission of Whole-Body Vertical Vibration from Seat-To-Head, Academic Press Inc, San Diego DA USA, 1989. 